\section{OP0099 EFFECTIVENESS OF REPETITIVE TRANSCRANIAL MAGNETIC STIMULATION IN PATIENTS WITH FAILED BACK SURGERY SYNDROME}

Canan Bursali ${ }^{1}$, Feyza Unlu Ozkan², Meryem Yilmaz Kaysin ${ }^{2}$, Nimet Dortcan ${ }^{3}$, ${ }^{2}$ Aktas Ilknur, Duygu Geler Kulcu ${ }^{4}{ }^{1}$ Aksaray University Training and Research Hospital, Physical Medicine and Rehabilitation, Aksaray, Turkey, ${ }^{2}$ University of Health Sciences, Fatih Sultan Mehmet Training and Research Hospital, Physical Medicine and Rehabilitation, Istanbul, Turkey, ${ }^{3}$ Istanbul Bilgi University, Faculty of Health Sciences, Istanbul, Turkey; ${ }^{4}$ University of Health Sciences, Haydarpasa Numune Training and Research Hospital, Physical Medicine and Rehabilitation, Istanbul, Turkey

Background: Failed back surgery syndrome (FBSS) is the term of persistent back and/or leg pain after lumbar surgery (1). Repetitive transcranial magnetic stimulation ( $r$-TMS) is a technique that allows non-invasive and relatively painless stimulation of cerebral cortex. It can reduce the experience of chronic pain by using magnetic field to produce small electrical currents in the cortex (2).

Objectives: The aim of this study is to determine the effectiveness of r-TMS treatment on patients with FBSS.

Methods: In this double-blinded, randomized, placebo-controlled trial, 20 patients (aged 34-65 years) clinically diagnosed as FBSS, who had a history of surgery for lumbar disk herniation with persistent back and leg pain. Only patients with no root compression in postoperative magnetic resonance imaging of lumbar spine were included. Patients were randomly assigned to r-TMS ( $n: 10)$ and sham (n:10) groups. Patients in r-TMS group received $5 \mathrm{~Hz}$ of $\mathrm{r}$-TMS, as a 20 -min (1000 pulses) daily session, 5 days per weeks, for a total of 10 sessions. R-TMS applied with MagVenture device (MagPro X100, Denmark, 2009) and figure eight coil (MMC 140 parabolic, MagVenture). Control group received sham r-TMS with the same protocol. Patients were assessed before and after the 1st and 5th sessions, at the end of the treatment and at 2 weeks, 1 month and 3 months after treatment. Visual Analogue Scale (VAS), DN-4, Oswestry Disability Index (ODI), Beck Depression Inventory (BDI), Pittsburgh Sleep Quality Index (PSQI) were used for evaluation.

Results: There were no statistically significant differences between the groups for age, sex, number of surgery, pain duration, working status and drug usage. Significant improvements were achieved in DN4, ODI, BDI and PSQI scores in rTMS group in comparison to sham group. Both groups displayed improvements in VAS scores while improvement in the sham group was limited to first month. Achieved improvements in r-TMS group in terms of VAS, DN4, ODI, BDI and PSQI scores were sustained at third month.

Conclusion: Repetitive transcranial magnetic stimulation might be an effective treatment alternative in patients with FBSS, further studies with larger groups are needed.

\section{REFERENCES:}

[1] Al Kaisy, A., Pang, D., Desai, M. J., Pries, P., North, R., Taylor, R. S.,. .. \& Rigoard, P. (2015). Failed back surgery syndrome: who has failed?. Neurochirurgie, 61, S6-S14.

[2] Johnson, S., Summers, J., \& Pridmore, S. (2006). Changes to somatosensory detection and pain thresholds following high frequency repetitive TMS of the motor cortex in individuals suffering from chronic pain. Pain, 123(12), 187-192.

Disclosure of Interests: None declared

DOI: 10.1136/annrheumdis-2019-eular.4289

\section{OP0100 1 THE EFFECT OF COMBINED PREGABALIN AND DULOXETINE IN FUNCTIONAL STATUS, QUALITY OF LIFE AND PSYCHOLOGICAL STATUS IN FIBROMYALGIA}

Samar Abd Alhamed Tabra*, Mohammed Hassan Abu-Zaid, Marwa Aboelhawa, Salwa Elmorsy. Faculty of Medicine Tanta University, Rheumatology and Rehabilitation, Tanta, Egypt

Background: Fibromyalgia syndrome (FMS) is a chronic pain syndrome that characterized by chronic widespread pain, muscle tenderness and is often accompanied by fatigue, sleep disturbance, and depressive moods ${ }^{1}$. Pregabalin and duloxetine are thought to reduce pain by different pharmacological actions, and the studies for combining different treatments in FMS are limited ${ }^{2}$.

Objectives: Assessment of the effectiveness of combined pregabalin and duloxetine in functional status, quality of life and psychological status in fibromyalgia Methods: One hundred and twenty adult patients of primary FMS (according to the 2010 ACR criteria for FMS) participated in this study. Patients with other source of chronic pain as inflammatory or degenerative rheumatic diseases, severe debilitating systemic diseases were excluded; also we excluded patients with any previous psychiatric disorders and patients who had any contraindications for the used treatments. After written consent; the patients were randomly divided into 4 equal groups; group I were given pregabaline (200 mg divided twice daily) for 6 months, group II were given duloxetine $(60 \mathrm{mg}$ divided twice daily) for 6 months, group III were given combined therapy ( $200 \mathrm{mg}$ divided twice daily of pregabaline plus $30 \mathrm{mg}$ daily single dose of duloxetine) for 6 months, group IV were given placebo treatment, any other previous treatments were stopped before starting this study. The patients were assessed at baseline, after 3 and 6 months of treatment by Fibromyalgia Impact Questionnaire (FIQ), Medical Outcomes Study Questionnaire Short Form 36 Health Survey (SF-36), Hospital Anxiety and Depression Scale (HADS) and serum beta endorphin.

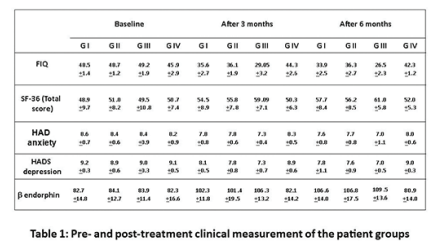

Results: In this study 115 patients completed the study. There was no significant difference between all groups in demographic data or educational status. At baseline there were no significant differences between all groups regarding FIQ, SF36 , HADS. Patients in all groups had low levels of $\beta$-Endorphin at baseline (82.7 $\pm 14.8,84.1 \pm 12.7,83.9 \pm 11.4$, and $82.3 \pm 16.6$ respectively). There was significant improvement $(\mathrm{P}<0.05)$ in FIQ, SF-36 and HADS in groups I, II and III after 3 and 6 months of treatment, while there was no significant improvement $(P>0.05)$ in group IV. There was significant better improvement $(P<0.05)$ in group III than in group I and II. The levels of serum $\beta$ - Endorphin were significantly elevated in the first 3 groups only $(P<0.05)$ with no significant difference between the 3 groups $(P>0.05)$. The results of the study are summarized in table 1 .

Conclusion: The combination therapy of pregabalin and duloxetine is more effective than monotherapy in treatment of fibromyalgia

\section{REFERENCES:}

[1] Egunsola O, Wylie CE, Chitty KM, Buckley NA. Systematic Review of the Efficacy and Safety of Gabapentin and Pregabalin for Pain in Children and Adolescents. Anesth Analg. 2018 Nov 16. doi: 10.1213/ ANE.0000000000003936. [Epub ahead of print]

[2] Gilron I, Chaparro LE, Tu D, Holden RR, et al. Combination of pregabalin with duloxetine for fibromyalgia: a randomized controlled trial. Pain. 2016 Jul;157(7):1532-40.

Disclosure of Interests: None declared

DOI: 10.1136/annrheumdis-2019-eular.170

\section{OP0101 COMPARATIVE EFFECTIVENESS OF LAND AND WATER-BASED EXERCISE ON QUALITY OF LIFE OF PATIENTS WITH FIBROMYALGIA: PRELIMINARY FINDINGS FROM THE AL-ÁNDALUS RANDOMISED CONTROLLED TRIAL}

Blanca Gavilán Carrera ${ }^{1}$, Víctor Segura-Jiménez ${ }^{2}$, Pedro Acosta-Manzano ${ }^{3}$ Fernando Estevez-Lopez ${ }^{1,4}$, Milkana Borges Cosic ${ }^{1}$, Virginia A Aparicio ${ }^{5}$, Ana Carbonell-Baeza 6 , Manuel Delgado-Fernández $1{ }^{1}$ Faculty of Sport Sciences, University of Granada, Department of Physical Education and Sports, Granada, Spain; ${ }^{2}$ Faculty of Education Sciences. University of Cádiz, Department of Physical Education, Granada, Spain; ${ }^{3}$ Faculty of Sport Sciences. University of Granada, Department of Physical Education and Sports, Granada, Spain; ${ }^{4}$ Ulster University, Belfast, United Kingdom; ${ }^{5}$ Faculty of Pharmacy. Faculty of Sport and Institute of Nutrition and Food Technology Sciences, Department of Physiology, Granada, Spain; ${ }^{6}$ Faculty of Education Sciences. University of Cádiz, Department of Physical Education, Cádiz, Spain

Background: Compelling evidence supports the efficacy of physical exercise in the management of fibromyalgia (FM) ${ }^{1}$. Although exercise interventions have typically focused on either land or water-based programs, research comparing the efficacy of both protocols is limited.

Objectives: The aim of this study was to compare the effect of two exercise interventions (land-based and water-based training) on quality of life ( $Q O L)$ of patients with FM.

Methods: Among the 272 participants initially randomized, a total of 151 (age:50.6 \pm 7.6 years, $98 \%$ women) completed all the assessments with an attendance $\geq 70 \%$ (land-based $n=48$, water-based $n=42$, control $n=61$ ). The intervention groups trained 3 non-consecutive days/week (60 minutes per session) during 24 weeks. Each session involved exercises to improve cardiorespiratory fitness, muscular strength, and flexibility. Physical and mental domains of QOL were assessed 